\title{
Numerical Symmetrization of State of Identical Particles
}

\author{
Oleg Utyuzh ${ }^{1}$, Grzegorz Wilk ${ }^{1}$, and Zbigniew Włodarczyk ${ }^{2}$ \\ ${ }^{1}$ The Andrzej Sottan Institute for Nuclear Studies, Hoża 69, 00-681 Warsaw, Poland \\ 2 Institute of Physics, Świętokrzyska Academy, Świętokrzyska 15, 25-405 Kielce, Poland
}

Received on 30 October, 2006; Revised 19 December, 2006

\begin{abstract}
The method of numerical symmetrization of state of identical particles proposed by us before is clarified and discussed.
\end{abstract}

Keywords: Bose-Einstein correlations; Statistical models; Fluctuations

\section{INTRODUCTION}

It is well known experimental fact that identical pions, which are produced in heavy ion collisions, being bosons show Bose-Einstein correlations. These correlations result from the quantum mechanical interference in the corresponding symmetrized $n$-particle wave function (where $n$ denotes the number of produced identical pions). They contain large amount of information about the statistical properties of the momentum and configuration space distribution of the system, and thus provide a potentially very useful method to probe the geometry of the hadronizing source (see, for example, [1] and references therein).

According to common understanding we are not able to determine which pion is emitted from which position in the source, so we are required by Bose statistics to add amplitudes for all possible alternate histories. In general, symmetrized wave function for $n_{\pi}$-pion state can be written in the following way [2]:

$$
\Psi_{\{p\}}(\{x\})=\frac{1}{\sqrt{n_{\pi} !}} \sum_{\sigma} \exp \left[-i \sum_{j=1}^{n_{\pi}} p_{j} r_{\sigma(j)}\right],
$$

where $\sigma(j)$ denotes the $j^{t h}$ element of a permutation of the sequence $\left\{1,2, \ldots, n_{\pi}\right\}$ and the sum over $\sigma$ denotes therefore the sum over all $n_{\pi}$ ! permutations in this sequence (dependence on positions of points of detection, which will vanish when calculating probabilities, was neglected). Here $r$ 's denote the points of production of secondaries. Because in the experiment one observes only momenta of produced secondaries these $r$ 's must be somehow get rid of. This is so far always done by integrating over $\left\{r_{j}\right\}$ with some assumed distribution $\rho\left(\left\{r_{j}\right\}\right)$, which is assumed to be factorizable and expressed by product of independent single particle distributions $\rho\left(\left\{r_{j}\right\}\right)=\prod_{j} \rho\left(r_{j}\right)[1,2]$. As result one gets the following probability of the $n_{\pi}$-pion state,

$$
\mathcal{P}_{1, ., n_{\pi}}=\frac{1}{n_{\pi} !} \sum_{\sigma} \prod_{j} \Phi_{j, \sigma(j)} \equiv \frac{1}{n_{\pi} !}\left\|\begin{array}{ccc}
\Phi_{1,1} & \cdots & \Phi_{1, n_{\pi}} \\
\vdots & \Phi_{j, j} & \vdots \\
\Phi_{n_{\pi}, 1} & \cdots & \Phi_{n_{\pi}, n_{\pi}}
\end{array}\right\|
$$

expressed by permanent of the matrix $\left\|\Phi_{i j}\right\|$, where

$$
\Phi_{i j}=\int e^{i\left(p_{i}-p_{j}\right) r} \rho(\{r\}) d^{4}\{r\} .
$$

For usually considered $n_{\pi}=2$ case one recovers well known classical expression for the probability of detecting two pions in the final state [1]:

$$
\mathcal{P}_{1,2}=\frac{1}{2}\left\|\begin{array}{ll}
\Phi_{1,1} & \Phi_{1,2} \\
\Phi_{2,1} & \Phi_{2,2}
\end{array}\right\|=\frac{1}{2}\left(\Phi_{1,1} \Phi_{2,2}+\Phi_{1,2} \Phi_{2,1}\right) .
$$

Unfortunately the execution time of direct computation of the permanent, eq.(1), grows exponentially with $n_{\pi}$ one has to devise some special methods like Metropolis procedure investigated in [2, 3] or von Neumann accepting-rejection method proposed in [4].

The Metropolis procedure proposed in [2] uses the standard Monte Carlo technique due to Metropolis. This is general method which allows to generate ensemble of $n$-body configurations according to some prescribed probability density. That is, the probability of a given configuration in the ensemble is precisely that given by the probability density used to generate "successive" configurations. In [2] this technique was used to modify directions of momentum vectors of number of selected particles from a system of $n$ identical particles in order to impose the $n$-particle distributions derived from BE correlation functions. This procedure is then repeated many times, changing selected particles, until a kind of "equilibrium" is achieved. As shown in [2] one was able in this way to generate typical multipion events which explicitly exhibit all correlations induced by Bose statistics. As a result of application of this algorithm a number of objects (called speckles) being clusters of large number of identical pions in the phase-space is being formed. The drawback of this method is that symmetrization of clusters with sizes (represented by the number of particles inside cluster, $n_{\text {cluster }}$ ) larger than $n_{\text {cluster }} \approx 10$ takes prohibitively long time. In [3] this method was therefore modified by limiting symmetrization only to particles in clusters. This was possible by using wave packets to describe produced particles instead of plane waves used in [2] allowing therefore for localization of particles within certain phase-space volume and for providing the suitable criterium for defining a cluster.

The accepting-rejection method investigated in [4] is based on the well known "hit-or-miss" technique of generating a set of random numbers according to a prescribed distribution. The method was designed to model collapse of a multiparticle wave function into a properly symmetrized state, as required by Bose quantum statistics. In contrast to the previous one it is sequential because $n$ multiparticle event is constructed by first choosing single particle in phase space, then adding to 
it the second one according to the assumed 2-particle correlation function $C_{2} \propto P_{2}$, then adding $3^{\text {th }}$ particle according to $C_{3} \propto P_{3}$ and so on. It is easy to realize that in this way one gets in the allowed phase space a "cell-like" structure because regions with some particles inside them already present will have bigger chance to attract new particle. In a sense it looks like follows: first particle forms a seed for a first cell. When second particle is added to event it can, depending on its distance from the first one, either remain in that cell or later on attract new particles and in this way start to form a new cell. This will then continue until all particles are used. Unfortunately, this sequential procedure is even more time consuming than the previous one.

\section{OUR PROPOSITION}

The above discussion shows that complexity of numerical symmetrization of wave function for all identical pions produced in a given event (cf., eq.(1)) can be substantially reduced if only one can justify the idea that such symmetrization should be applied to groups of limited number of particles, as proposed in [3]. As argued there this can be achieved by dividing, according to some prescribed procedure, the initial set of secondaries into clusters consisting of identical particles with similar momenta. Such groups of particles, but this time of (almost) equal energies, were introduced in [5] under the name Elementary Emitting Cells (EEC's). The physical justification was that bosonic particles, because of their statistical properties, tend to occur, as much as possible, in the same state. One should realize now that such decomposition corresponds to factorization of permanent given by eq.(2) into matrix with a block structure:

$$
\left\|\begin{array}{ccc}
\Phi_{1,1} & \cdots & \Phi_{1, n_{\pi}} \\
\vdots & \Phi_{i, i} & \vdots \\
\Phi_{n_{\pi}, 1} & \cdots & \Phi_{n_{\pi}, n_{\pi}}
\end{array}\right\| \Rightarrow\left\|\begin{array}{ccc}
E E C_{1} & \cdots & 0 \\
\vdots & \ddots & \vdots \\
0 & \cdots & E E C_{N_{\text {cell }}}
\end{array}\right\|
$$

This idea of EEC was exploited by us further in [6] where instead of symmetrization of multiparticle wave function (depending on space-time positions, $x$, and energy-momenta, $p$ ) we worked in the number of particles basis. The bosonic character of the produced secondaries means in this case the specific bunching of identical particles in the phase space. In fact it is nothing else but modelling the correlations of fluctuations due to quantum statistics present in the system, which for 2particle case are represented by:

$$
\left\langle n_{1} n_{2}\right\rangle=\left\langle n_{1}\right\rangle\left\langle n_{2}\right\rangle+\rho \sigma\left(n_{1}\right) \sigma\left(n_{2}\right)
$$

$\sigma(n)$ is dispersion of the multiplicity distribution $P(n)$ and $\rho$ is the correlation coefficient depending on the type of particles produced: $\rho=+1,-1,0$ for bosons, fermions and Boltzmann statistics, respectively). The important feature of the EEC method is that number of particles in each cell follows by definition the geometrical (or Bose-Einstein) distribution characterized for identical bosonic particles.

It should be mentioned at this point that the importance of bunching of particles in modelling quantum statistical effects has been demonstrated already in [7]. In this paper, following
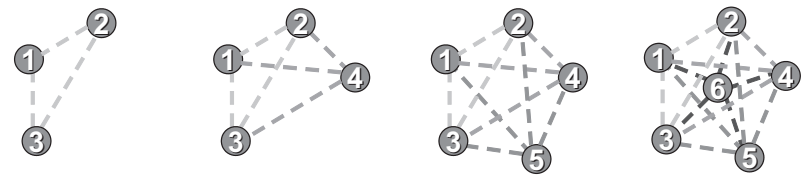

FIG. 1: Representation of algorithm for the sequential model, eq. (9).

ideas of information theory, authors constructed Monte-Carlo (MC) event generator for multiparticle production processes (and applied it to $e^{+} e^{-}$reactions). The main point was the assumption that particles of the same charge are located in cells (in their case they were constructed in rapidity space and were of equal size). It turned out that such division of phase space into cells was crucial for obtaining the characteristic form of the 2-body BEC correlation function $C_{2}\left(Q=\left|p_{i}-p_{j}\right|\right)$ (i.e., the one peaked and greater than unity at $Q=0$ and then decreasing in a characteristic way towards $C_{2}=1$ for large values of $Q$, out of which one usually deduces the spatiotemporal characteristics of the hadronization source [1]).

In our case we argue that the method of production of EEC proposed in [5] can be used to define the structure of clusters obtained in [3] (with, as it turns out, about $n_{c l}=2$ particles per cluster on average, depending on circumstances [8]). Therefore, instead of symmetrizing all particles in a given event, one symmetrizes separately particles in a number of EEC's with $n_{c l} \ll n_{\pi}$. In the plane wave approximation used in [2], one has therefore for some typical EEC the following $n_{c l}$-particle probability function:

$$
\mathcal{P}_{1, \ldots, n_{c l}}^{[n]}=1+\frac{2}{n_{c l} !} \sum_{\sigma^{\prime}>\sigma=1}^{n_{c l} !} \cos \left\{\sum_{j=1}^{n_{c l}} p_{i}\left[r_{\sigma(j)}-r_{\sigma^{\prime}(j)}\right]\right\} .
$$

Notice that eq. (7) still contains (unmeasurable) positions of production of particles, $\left\{r_{\sigma(j)}\right\}$. Therefore they will be later eliminated by selecting them from some assumed distribution in a kind of numerical integration process. It corresponds to analytical integrations encountered before but in our approach we do not limit in any way the form of density function used (it had to be factorizable before). It should be stressed that when all particles in the cluster are exactly in the same state then one gets, as expected,

$$
\left.\mathcal{P}_{1, \ldots, n_{c l}}^{[n]}(\max )\right|_{p_{1}=\cdots=p_{n_{c l}}}=n_{c l} ! .
$$

However, in practice, even now from time to time one encounters EEC with $n_{c l} \gg 1$, in which case eq.(7) is still very time consuming. For such cases we have to use some approximate schemes. The first one is sequential (cf. Fig. 1): one starts with some single particle in EEC and adds new particles one by one to all others already present and correlate them by using standard 2-particle probability: $1+\cos (\delta r \cdot \delta p)$. The resulting probability to get $n_{c l}$-particle state is given by:

$$
\mathcal{P}_{1, \ldots, n_{c l}}^{[2]}=\mathcal{P}_{1, \ldots, n_{c l}-1} \cdot \prod_{j=1}^{n_{c l}-1}\left[1+\cos \left(\delta r_{j n_{c l}} \cdot \delta p_{j n_{c l}}\right)\right] .
$$

Although appealing (in fact it resembles procedure used in [4]) it has drawback that when all particles are in the same state then

$$
\left.\mathcal{P}_{1, \ldots, n_{c l}}^{[2]}(\max )\right|_{p_{1}=\cdots=p_{n_{c l}}}=2^{\frac{1}{2} n_{c l}\left(n_{c l}-1\right)}
$$




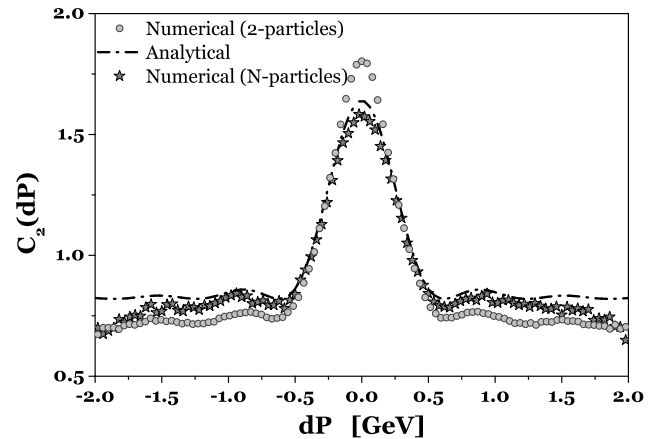

FIG. 2: Example of $C_{2}$ modelled for single EEC with $N=4$ by using 2-particles (sequential) correlations as given by eq. (9) (circles) and full $N$-particles correlations as given by eq. (7) (stars). For comparison, the analytical result, $C_{2}(\delta P)=1+\sin ^{2}(R \delta P) /(R \delta P)^{2}$, obtained for uniform one dimensional source in space with $R=1 \mathrm{fm}$ is also shown (dotted-dashed line).

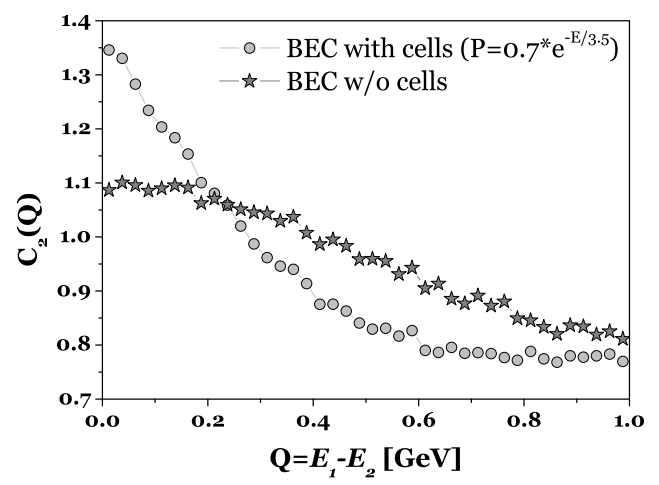

FIG. 3: Comparison of $C_{2}$ modelled by using MC event generators with EECs (circles) and without EECs but with particles selected directly from the corresponding Bose-Einstein distribution [9]. In both cases as reference event the Boltzmann distribution was used.

i.e., for $n_{c l}>2$ the correlations are stronger and the maximal value of probability function gets bigger than allowed limit defined by eq.(8). Both, exact (7) and sequential (9) methods are compared in Fig. 2. Approximation (9) keeps the width of $C_{2}$ the same, however it differs substantially in $C_{2}(\delta P=0)$ (from which one tends to estimate the so called chaoticity of the hadronizing source [1]). The other possible approximation, which was used in our hitherto applications [8], is that all particles in a given EEC are correlated only with the first particle defining this cell, not between themselves. It is interesting to notice that the results for $C_{2}$ obtained this way are almost the same as those obtained by using the full method, eq. (7), but differ from that of eq. (9).

We close this section by noticing recent attempt to imitate the bosonic nature of particles produced by MC event generator, in which they were chosen directly according to BoseEinstein distribution [9]. This method seems to be very natural and occurred to be also very fast. However, as one can see from Fig. 3, where it is compared with our approach, it leads to completely different $C_{2}$ substantially underestimating the correlation function $C_{2}$. We claim therefore that such procedure inevitably loses some piece of important information, namely the fact that EEC's are formed and that Bose-Einstein distributions are only for particles in such EEC's, not in the whole event. In fact, signal of possible Bose-Einstein correlations without cells is seen in Fig. 3 are the trivial correlations, which can be eliminated by the proper choice of the reference event.

\section{SUMMARY}

We would like to summarize by stressing that there is no way to add to any of the existing MC event generators effects of quantum statistics, in particular Bose-Einstein one (BE). This is because they are all build on basis of classical physics with both the space-time and energy-momentum characteristics of produced secondaries used simultaneously. The only way out advocated here (albeit, most probably, not very practical one and therefore hardly to be followed) is to build the multiparticle $\mathrm{MC}$ event generator ab initio, with $\mathrm{BE}$ properties (like bunching in phase space) being one of its basic principles and consisting its first step. All other features of such generator would have to be added only after this. So far there is only one working example of this type [7], our efforts [8] aim for its further generalization and will be continued.

\section{Acknowledgements}

OU is grateful for support and for the warm hospitality extended to him by organizers of the ISMD2006. Partial support of the Ministry of Science and Higher Education (grants $\mathrm{Nr}$ 621/E-78/SPB/CERN/P-03/DWM 52/2004-2006 and 1 P03B 02230 ) is acknowledged (OU and GW).
[1] T. J. Humanic, Int. J. Mod. Phys. E 15, 197 (2006).

[2] W. A. Zajc, Phys. Rev. D 35, 3396 (1987).

[3] H. Merlitz and D. Pelte, Z.Phys. A 351, 187 (1995); Z. Phys. A 357, 175 (1997).

[4] J. G. Cramer, Event Simulation of High-Order Bose-Einstein and Coulomb Correlations, Univ. of Washington preprint (1996, unpublished).

[5] M. Biyajima, N. Suzuki, G. Wilk, and Z. Włodarczyk; Phys. Lett. B 386, 297 (1996).

[6] O. V. Utyuzh, G. Wilk and Z. Włodarczyk, Phys. Lett. B 522, 273 (2001) and Acta Phys. Polon. B 33, 2681 (2002) (hep- $\mathrm{ph} / 0205087)$

[7] T. Osada, M. Maruyama, and F. Takagi, Phys. Rev. D 59, 014024 (1999).

[8] O. V. Utyuzh, G. Wilk, and Z. Włodarczyk, hep-ph/0503046, Acta Phys. Hung. A - Heavy Ion Phys. 25 (1), 83 (2006); hepph/0509320, AIP Conf. Proc. 828, 75 (2006); hep-ph/0509342, to be published in Nukleonika 51 (Supplement 3) (2006).

[9] A. Kisiel, T. Tałuć, W. Broniowski, and W. Florkowski, Comp. Phys. Comm. 174, 669 (2006). 